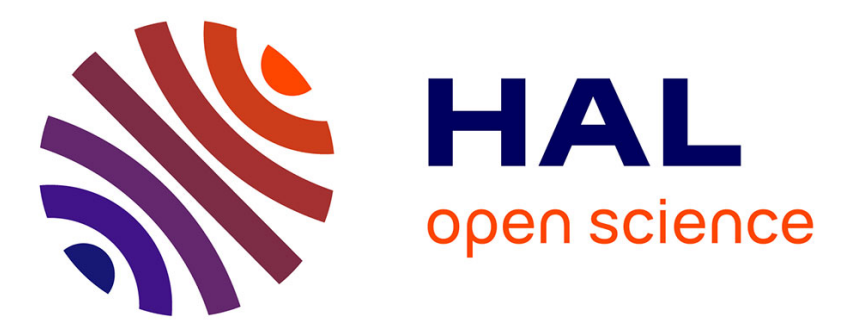

\title{
Acoustic and streaming velocity components in a resonant waveguide at high acoustic levels
}

\author{
Virginie Daru, Ida Reyt, Hélène Bailliet, Catherine Weisman, Diana
}

Baltean-Carles

\section{- To cite this version:}

Virginie Daru, Ida Reyt, Hélène Bailliet, Catherine Weisman, Diana Baltean-Carles. Acoustic and streaming velocity components in a resonant waveguide at high acoustic levels. Journal of the Acoustical Society of America, 2017, 141, pp.563-574. 10.1121/1.4974058 . hal-02390553

\section{HAL Id: hal-02390553 \\ https://hal.science/hal-02390553}

Submitted on 3 Dec 2019

HAL is a multi-disciplinary open access archive for the deposit and dissemination of scientific research documents, whether they are published or not. The documents may come from teaching and research institutions in France or abroad, or from public or private research centers.
L'archive ouverte pluridisciplinaire HAL, est destinée au dépôt et à la diffusion de documents scientifiques de niveau recherche, publiés ou non, émanant des établissements d'enseignement et de recherche français ou étrangers, des laboratoires publics ou privés. 


\title{
Acoustic and streaming velocity components in a resonant waveguide at high acoustic levels
}

\author{
Virginie Daru ${ }^{\text {a) }}$ and Ida Reyt \\ Arts et Métiers ParisTech - Lab. DynFluid - 151 bd de l'Hôpital 75013 Paris, LIMSI, CNRS, F-91405 Orsay, \\ France \\ Hélène Bailliet \\ Institut Pprime, CNRS - Université de Poitiers - ENSMA, ENSIP, 6 rue Marcel Doré, Bâtiment B17 - BP 633, \\ 86022 Poitiers Cedex, France
}

\begin{abstract}
Catherine Weisman and Diana Baltean-Carlès
Sorbonne Universités, UPMC Univ Paris 06, UFR d'Ingénierie, 4 Place Jussieu, 75252 Paris Cedex 05, LIMSI, CNRS, F-91405 Orsay, France
\end{abstract}

(Received 24 August 2016; revised 14 December 2016; accepted 2 January 2017; published online 26 January 2017)

\begin{abstract}
Rayleigh streaming is a steady flow generated by the interaction between an acoustic wave and a solid wall, generally assumed to be second order in a Mach number expansion. Acoustic streaming is well known in the case of a stationary plane wave at low amplitude: it has a half-wavelength spatial periodicity and the maximum axial streaming velocity is a quadratic function of the acoustic velocity amplitude at antinode. For higher acoustic levels, additional streaming cells have been observed. Results of laser Doppler velocimetry measurements are here compared to direct numerical simulations. The evolution of axial and radial velocity components for both acoustic and streaming velocities is studied from low to high acoustic amplitudes. Two streaming flow regimes are pointed out, the axial streaming dependency on acoustics going from quadratic to linear. The evolution of streaming flow is different for outer cells and for inner cells. Also, the hypothesis of radial streaming velocity being of second order in a Mach number expansion, is not valid at high amplitudes. The change of regime occurs when the radial streaming velocity amplitude becomes larger than the radial acoustic velocity amplitude, high levels being therefore characterized by nonlinear interaction of the different velocity components. c○2017 Acoustical Society of America.
\end{abstract}

\section{INTRODUCTION}

Acoustic streaming is a mean flow generated by Reynolds stresses in a fluid that supports an acoustic wave, either due to absorption in the main body of an irrotational sound beam (for Eckart streaming or Quartz wind), or associated to Stokes boundary layer adjacent to a solid boundary. This second type of streaming is usually referred to as Rayleigh streaming because Lord Rayleigh developed a solution that describes the steady vortices generated in the core of the fluid (also called outer cells) in a wide channel. ${ }^{1}$ Rayleigh's analysis applied to the case of a large cylindrical guide yields

$$
\begin{aligned}
& u_{2, \text { Rayleigh }}=-\frac{3 U_{\mathrm{ac}}^{2}}{8 c} \sin (2 k x)\left[1-2 \eta^{2}\right], \\
& v_{2, \text { Rayleigh }}=\frac{3 U_{\mathrm{ac}}^{2}}{8 c} k R \cos (2 k x)\left[\eta-\eta^{3}\right],
\end{aligned}
$$

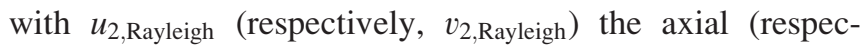
tively, radial) component of streaming velocity, $U_{\mathrm{ac}}$ the

\footnotetext{
a)Electronic mail: daru@limsi.fr
}

acoustic velocity amplitude at its antinode, $c$ the speed of sound, $R$ the channel radius, $x$ the axial coordinate, $k$ the complex wave number, $\eta=r / R$ the radial coordinate with $r$ the distance from guide axis. In order to obtain these equations, terms in $e^{-R(1-\eta) / \delta_{\nu}}$ were neglected in the complete equations of Rayleigh solution, which is valid far enough from the boundary layer (with $\delta_{\nu}=\sqrt{2 \nu / \omega}$ the acoustic boundary layer thickness, $\omega$ being the angular frequency). These equations clearly show the main characteristics of Rayleigh streaming: Streaming velocities are of second order in powers of acoustic Mach number, with vortices that span $\lambda / 4$; axial and radial streaming components have quite different axial and radial dependencies. Subsequently, Schlichting ${ }^{2}$ first described the inner streaming vortices confined to the boundary layer and rotating in the opposite direction than that of the Rayleigh outer cells, under the hypothesis of incompressible flow. Later, $\mathrm{Qi}^{3}$ included the fluid compressibility and temperature effects to improve the description of inner streaming. Rott ${ }^{4}$ improved the description of the outer streaming by taking into account thermal effects. Experimental studies were started later than theoretical ones, using second order time average pressure measurements ${ }^{5}$ or making profit of the development of laser velocimetry techniques (e.g., Ref. 6). 
The above-described characteristics of Rayleigh streaming are modified when the acoustics are increased to high levels. Available studies focused on the description of high level axial component of the streaming velocity. Thompson and Atchley ${ }^{7}$ first observed that the variation of the axial component of streaming velocity, with respect to the transverse coordinate, departs from a parabola and that its axial dependence departs from a pure sinusoidal function at high acoustic amplitudes. Moreau et al. ${ }^{8}$ further described these distortions; they characterized the change of regime of the axial component of the streaming velocity from parabolic Rayleigh theory and found that for $\mathrm{Re}_{\mathrm{NL}}>20$ axial streaming velocity is not in agreement with any available theory [see Eq. (3) for the definition of $\mathrm{Re}_{\mathrm{NL}}$ ]. Then it was found both experimentally ${ }^{9}$ and numerically ${ }^{10}$ that when the acoustic level is further increased, the previously described distortion of centerline axial streaming velocity towards acoustic velocity nodes leads to the generation of counter-rotating additional vortices near the acoustic velocity antinodes. This kind of behaviour was found to be followed by outer streaming cells only; results in the near wall region show that inner streaming vortices are only slightly modified.

The investigation of the origins of the mutation of streaming at high amplitudes has already been considered in the literature. The effect of inertia on streaming was the first phenomenon suspected to lead to high level deformation of streaming. Menguy and Gilbert ${ }^{11}$ introduced the nonlinear Reynolds number,

$$
\operatorname{Re}_{\mathrm{NL}}=\left(\frac{U_{\mathrm{ac}}}{c}\right)^{2}\left(\frac{R}{\delta_{\nu}}\right)^{2}
$$

that compares inertia of streaming and viscosity and determines the degree to which nonlinear effects of fluid inertia become dominant. This nonlinear Reynolds number has then been commonly used as criteria to range the conformity of streaming to Rayleigh pattern, the case $\mathrm{Re}_{\mathrm{NL}} \ll 1$ being referred to as "slow streaming" and the case $\operatorname{Re}_{\mathrm{NL}} \geq 1$ as "nonlinear streaming" or "fast streaming." All available analytical models are restricted to slow streaming (e.g., Ref. 12). The effect of inertia on streaming was further analyzed by Daru et al., ${ }^{13}$ thanks to the development of an averaged model simulation that permitted to isolate inertia from other high level effects. This model showed that the distortion associated with inertia of streaming flow is opposite to the one observed both experimentally [by laser Doppler velocimetry (LDV)] and numerically [with direct numerical simulations (DNS)] for streaming at high level: Inertia is associated with a distortion of streaming towards the acoustic velocity antinodes, making impossible the emergence of new streaming cells. This allowed us to identify the effect of inertia in the establishment of acoustic streaming and to assert that it is not the leading phenomena in the evolution of Rayleigh cells at high amplitudes.

Other effects have been considered as possibly responsible for the mutation of streaming at high amplitudes: ${ }^{10}$ Nonlinear propagation and thermal effects associated with acoustically induced heat transfer in a standing waveguide.
However, it was also shown that even if these phenomena do influence the streaming pattern at high levels, neither can be considered as being at the origin of the change of regime for Rayleigh vortices. Therefore, it was necessary to go further into the investigation of the origins of this mutation. In the present paper, we decided to investigate the possible nonlinear interaction between streaming and acoustics. The method of successive approximations is usually applied in acoustics and in particular, when approaching acoustic streaming. It implies that the different order components (including the acoustic first order and the streaming second order components) do not interact with each other. The aim of this paper is to evaluate the validity of this hypothesis when the acoustic amplitude is increased. To this effect, numerical and experimental results are gathered in order to further describe the modifications of streaming and acoustics at high acoustic amplitudes. A description of the usually considered axial velocity components for streaming and acoustics from low to high level regimes is proposed together with a description of the less considered radial velocity components, again for streaming and acoustics. The evolution of the outer streaming velocity amplitude $U_{s}$ with the acoustic amplitude $U_{\text {ac }}$ is further described. An important change is noticed: $U_{s}$ is found to go from a quadratic to a linear dependence on $U_{\mathrm{ac}}$. Attention is paid to both outer and inner streaming and it is shown that the variation of streaming velocity amplitude with acoustic velocity amplitude does not occur similarly for the two kinds of streaming. It is shown that the hypothesis of streaming velocity being much smaller than acoustic velocity is restrictive at high amplitudes for the radial velocities.

The paper is organized as follows. Section II presents the experimental setup and the numerical method and procedure. In Sec. III, the evolution of axial components of acoustic and streaming velocities are described from low to high amplitudes. The change of regime from quadratic to linear dependence of the axial streaming velocity on the acoustic amplitude is analyzed. In Sec. IV, the evolution of radial components of acoustic and streaming velocities is discussed from low to high amplitudes and the nonlinear interaction of radial acoustic and streaming velocities is investigated.

\section{METHODS AND CONFIGURATIONS}

As stated above, both experimental and numerical studies are used in this paper to obtain a thorough description of the evolution of streaming from low to high acoustic levels. In both these approaches, a cylindrical tube of length $L$ and radius $R$ is considered. The tube is filled with air at atmospheric pressure, in which a high level standing wave is generated by exciting a resonant mode.

Because we want to focus on one among other high level phenomena that can be responsible for the evolution of Rayleigh streaming, it is necessary to separate the contribution of the different phenomena encountered at high level, or at least to scale them. Thompson et al. ${ }^{14}$ first showed that temperature gradients influence Rayleigh streaming. In the experimental setup, the thermal condition corresponded to the "uncontrolled" condition as defined by Ref. 14, that is, the resonator was exchanging heat with the surrounding air 
and a small temperature gradient was created. In the numerical simulations isentropic acoustic oscillations are considered so that thermal variations are not taken into account and the energy equation is not considered.

Shock wave formation can also influence Rayleigh streaming. In the experimental setup, a cylindrical guide is connected to loudspeakers via convergents; the resulting coupled resonant system offers the advantage that its modes are not in a harmonic series, preventing the cascade of energy from $\omega$ (the angular frequency of the acoustic wave) to its harmonics by nonlinear propagation. This is not the case of the configuration studied numerically, where shock waves can develop (as shown in Ref. 10), but we have checked that they are of small intensity and do not have drastic influence on streaming cells, during the physical time corresponding to numerical simulations ( $\approx 200$ periods).

In the following, the experimental setup and procedure is first described and then the numerical calculation is presented. Finally the experimental and numerical data set selected for analysis are given.

\section{A. Experimental setup}

The setup used to observe the acoustic streaming phenomenon is shown in Fig. 1. The cylindrical tube, with $R=19.5 \mathrm{~mm}$, is connected at each end to a loudspeaker via connecting tubes, designed to avoid separation effects related to the singularities in change of section. Either the first mode corresponding to $L_{\exp }=\lambda / 2$ or the third mode corresponding to $L_{\exp }=3 \lambda / 2$ is excited. The total length of the waveguide is $L_{\exp }=2.13 \mathrm{~m}$ so that the two distinct resonance frequencies are 88 and $240 \mathrm{~Hz}$.

Wood smoke is used as seeding particles in order to perform particle velocity measurements by Laser. LDV allows us to access the axial particle velocity and particle image velocimetry (PIV) allows us to access the streaming velocity field in a $(x, r)$ plane.

The single component LDV system is a Dantec Dynamic model 2580; the probe is mounted on a three-axis positioning system. The argon krypton laser has an optical wavelength of $514.5 \mathrm{~nm}$ and a power of $25 \mathrm{~W}$. The parameters of LDV system are adjusted for axial particle velocity measurements and the axial streaming velocity is deduced from these measurements. Moreau et al. ${ }^{8}$ showed that in such a setup it is necessary to wait for 26 min after the acoustic field is switched on to ensure that the streaming field is fully established. Also they found that a good compromise between a sufficient number of measured tracer particles per measuring point for a precise streaming velocity estimate and a short enough measuring time that insures a correct seeding during a whole scan is obtained when choosing to acquire either 70000 points or to stop acquisition after $10 \mathrm{~s}$.

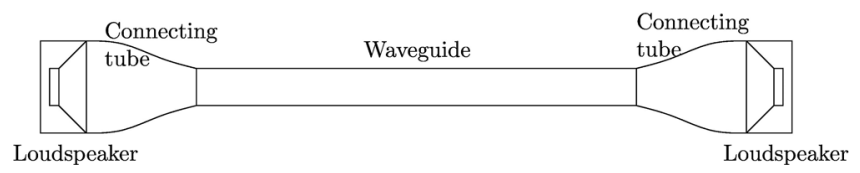

FIG. 1. Diagram of the experimental apparatus.
The results presented in the following were obtained with these settings.

On the other hand, PIV measurements were performed by generating a laser sheet of $1 \mathrm{~mm}$ thickness with a pulsed laser (Quantel Mini-Yag) having a $532 \mathrm{~nm}$ wavelength, issuing a pulsed energy of $30 \mathrm{~mJ}$ and synchronized with a maximum frequency of $20 \mathrm{~Hz}$. To insure convergence of the streaming velocity field calculation, 500 pairs of pictures were recorded by a JAI RM-4200CL camera (maximum of 15 pictures/s, $2048 \times 2048$ pixels) and the 500 phase locked velocity fields obtained were averaged. This installation allows the discretization of the velocity field with resolution along the guide axis/radius $\Delta x=\Delta r=0.52 \mathrm{~mm}$. For more information on the evaluation of the streaming velocity see the work of Reyt et al. ${ }^{9}$

\section{B. Numerical method}

The configuration numerically studied consists in a fluid oscillating as the result of an acoustic standing wave initiated by shaking a resonator along its axial direction at $\omega$. Resonant conditions are imposed and the $L=\lambda / 2$ mode is excited. The flow is modeled with the compressible NavierStokes equations expressed in the moving frame attached to the tube, so that a forcing source term is added. Because the wave propagation is considered as isentropic (in order to approach the experimental "uncontrolled" thermal condition), the energy equation is decoupled from the other equations and we only solve the mass and momentum equations. The equations are numerically solved with an axisymmetric formulation using high order finite difference schemes. ${ }^{10,15}$

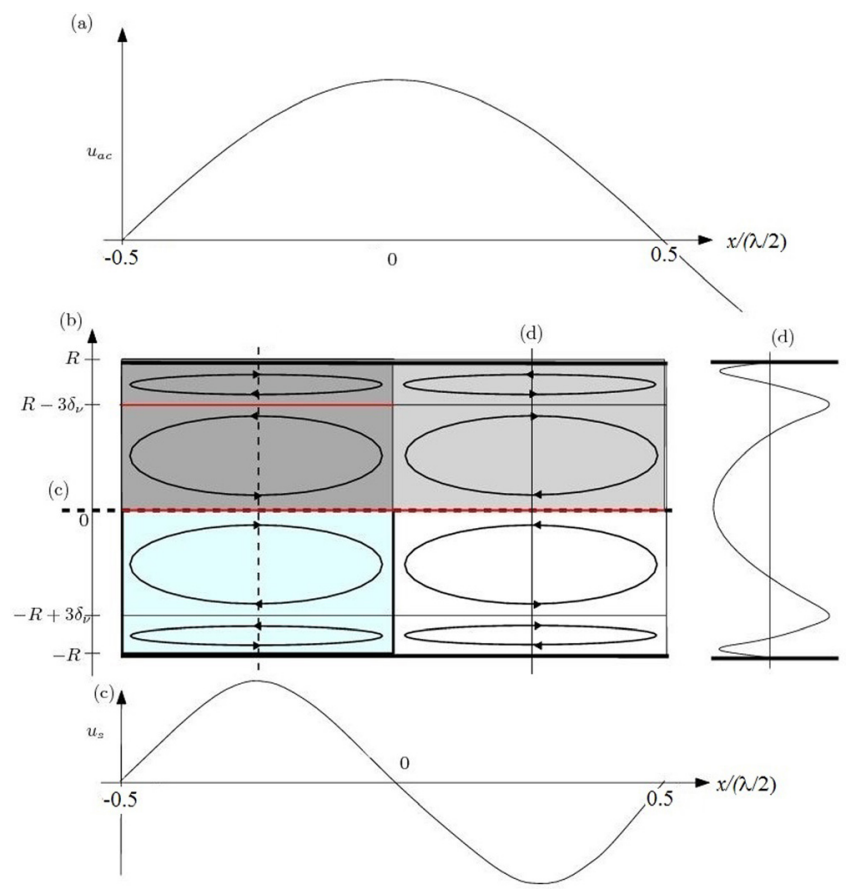

FIG. 2. (Color online) (a) Acoustic wave pattern. (b) Schematic representation of the corresponding slow streaming velocity field. Grey and dark grey zone: simulation domain. Blue and dark grey zone: PIV measurements. Red lines: LDV measurements. (c) Variation of axial streaming velocity with respect to the axial coordinate at $r=0$. (d) Variation of axial streaming velocity with respect to the radial coordinate at $x=\lambda / 8$. 
TABLE I. Parameters of the different presented cases.

\begin{tabular}{|c|c|c|c|c|c|c|}
\hline Name & $f(\mathrm{~Hz})$ & $R / \delta_{\nu}$ & $R / \lambda$ & $U_{a c}(\mathrm{~m} / \mathrm{s})$ & $U_{s}(\mathrm{~m} / \mathrm{s})$ & $\operatorname{Re}_{\mathrm{NL}}$ \\
\hline$N_{1}$ & 20000 & 20 & $2 \times 10^{-3}$ & {$[0.2-60]$} & {$\left[0.4 \times 10^{-4}-2.4\right]$} & {$\left[1.3 \times 10^{-4}-12.18\right]$} \\
\hline $\mathrm{N}_{2}$ & 20000 & 40 & $3.7 \times 10^{-2}$ & {$[0.4-80]$} & {$\left[1.6 \times 10^{-4}-4.9\right]$} & {$\left[2 \times 10^{-3}-86.62\right]$} \\
\hline$N_{3}$ & 20000 & 50 & $4.5 \times 10^{-2}$ & {$[0.5-73]$} & {$\left[2.6 \times 10^{-4}-4.2\right]$} & {$\left[5.3 \times 10^{-3}-112.7\right]$} \\
\hline$N_{4}$ & 5000 & 50 & $2.25 \times 10^{-2}$ & {$[0.5-62]$} & {$\left[2.6 \times 10^{-4}-3.3\right]$} & {$\left[5.3 \times 10^{-3}-81.3\right]$} \\
\hline$E_{1}$ & 88 & 84 & $5 \times 10^{-3}$ & {$[2.7-45]$} & {$\left[90 \times 10^{-4}-1.5\right]$} & {$[0.43-120.87]$} \\
\hline$E_{2}$ & 240 & 138 & $1.4 \times 10^{-2}$ & {$[2.7-30]$} & {$\left[80 \times 10^{-4}-0.4\right]$} & {$[1.17-145]$} \\
\hline
\end{tabular}

The physical boundary conditions in the moving frame are: no slip on the boundary walls parallel to horizontal symmetry axis, and symmetry conditions on the vertical boundaries. The instantaneous flow is calculated with a DNS approach and the mean flow is obtained by time averaging over an acoustic period. All results presented below are obtained using a regular mesh of rectangular cells composed of 500 points along the axis, and of $5 \times R / \delta_{\nu}$ points in the radial direction. The time step $\delta t$ is fixed in order to insure stability of the numerical scheme $\left(\delta t=8 \times 10^{-9} \mathrm{~s}\right.$ for $N_{1}, N_{2}, N_{3}$, $\delta t=1.6 \times 10^{-8} \mathrm{~s}$ for $N_{4}$, the different numerical configurations being defined in Sec. II C).

\section{Studied configurations}

A schematic representation of streaming expected in the acoustic resonators under study is given in Fig. 2. The domain considered for numerical simulations is shown in grey. LDV scans correspond to the horizontal red lines (along the resonator axis and in the near wall region) and the PIV measurement domain is depicted by the light blue/dark grey zone. Figure 2 recalls that in the outer cells (located in the resonator core) the flow is circulating from the acoustic velocity nodes towards the acoustic velocity antinodes along the central axis and returns in the vicinity of the wall to complete a closed loop. The location $r=R-3 \delta_{\nu}$ corresponds to the limit between the two streaming cells (inner cell and outer cell), so that the amplitude of the axial streaming velocity has a local maximum [Fig. 2(d)] which will be referred to as the inner streaming amplitude in the following.

In this study we compare results from two experimental (noted $E_{1}$ and $\left.E_{2}\right)$ and four numerical $\left(N_{1}\right.$ to $\left.N_{4}\right)$ configurations. The corresponding parameters are summarized in Table I. Note that for the chosen configurations, $20 \leq R / \delta_{\nu}<140$, and thus the inner vortices fill a much smaller portion of the guide than in Fig. 2(b) where the represented guide is narrow $\left(R / \delta_{\nu}\right.$ is about 4$)$. For the large guides under study, Eqs. (1) and (2) provide a good description of outer streaming cells at low acoustic amplitudes. The range of variation of $U_{a c}$ which is the amplitude of the axial acoustic velocity at antinode, shown in Table I, is chosen to encompass a variety of streaming flows in order to exhibit the different flow regimes (there are between 6 and 19 data points covering the range of $U_{\mathrm{ac}}$ values). Because the size of the numerical system is very different from the size of the experimental system (to reduce computational time), working frequencies are very different. Nondimensional analysis, ${ }^{10}$ however, shows that the same flow behaviour is expected if $M \ll 1, \operatorname{Re} \gg 1$ and $S h \ll 1$, where $M=U_{\mathrm{ac}} / c, \operatorname{Re}=1 / 2\left(\lambda / \pi \delta_{\nu}\right)^{2}, S h=\delta_{\nu} / R$. These conditions are fulfilled by all experimental and numerical configurations under study.

In this paper we want to check if the oscillation part and the mean part (of different order of magnitude) of the particle velocity can be considered as independent from each other. For this purpose we consider the two particle velocity components: axial and radial and we consider the evolution of the contribution of acoustics and streaming to these components as the acoustic level is increased. Several strategic positions are chosen for discussing these evolutions, summarized in Table II.

\section{AXIAL ACOUSTIC AND STREAMING VELOCITIES FROM LOW TO HIGH LEVELS}

\section{A. Mutation of streaming at high level}

The characteristics of Rayleigh streaming described by Fig. 2 and Eq. (1) are also observed on Fig. 3, top-left, that shows contours of the axial streaming velocity resulting from the averaged PIV measurements for the experimental data set $E_{2}$ at relatively low level. The limit between two streaming cells is shown by thick vertical dotted lines. Due to the fact that the guide is much larger than in Fig. 2(b), only the Rayleigh outer cells are visible. Similarly, Fig. 3, top-right, representing the contours of the axial streaming velocity together with velocity vectors of the streaming flow calculated from $N_{3}$ numerical simulations, show results in agreement with the above description of slow streaming. In Fig. 3 top, one can see that the shape of the outer cells is not perfectly elliptic as it is the case in the schematic representation given by Fig. 2; the center of a cell is located approximately at $r / R=0.7$, in agreement with Eq. (1).

TABLE II. Spatial evolution of the velocity components considered.

\begin{tabular}{lccc}
\hline \hline Component & Axial evolution & Radial evolution & Section \\
\hline Axial acoustics & $u_{\mathrm{ac}}(x, r=0)$ & $u_{\mathrm{ac}}(x=\lambda / 8, r)$ & III B \\
& $u_{\mathrm{ac}}\left(x, r=R-2 \delta_{\nu}\right)$ & & III B \\
Axial streaming & $u_{s}(x, r=0)$ & $u_{s}(x=\lambda / 8, r)$ & III B \\
& $u_{s}\left(x, r=R-2 \delta_{\nu}\right)$ & & III B \\
Radial acoustics & & $v_{\mathrm{ac}}(x=-\lambda / 4+3 \lambda / 100, r)$ & IV A \\
& & $v_{\mathrm{ac}}(x=-\lambda / 4+7 \lambda / 100, r)$ & IV A \\
& & $v_{\mathrm{ac}}(x=-\lambda / 4+11 \lambda / 100, r)$ & IV A \\
& & $v_{\mathrm{ac}}(x=-\lambda / 4+15 \lambda / 100, r)$ & IV A \\
& & $v_{\mathrm{ac}}(x=-\lambda / 4+19 \lambda / 100, r)$ & IV A \\
& & $v_{\mathrm{ac}}(x=-\lambda / 4+23 \lambda / 100, r)$ & IV A \\
Radial streaming & $v_{s}(x, r=0.564 R)$ & $v_{s}(x=0, r)$ & IV A \\
& & $v_{s}(x=\lambda / 2, r)$ & IV A \\
\hline \hline
\end{tabular}



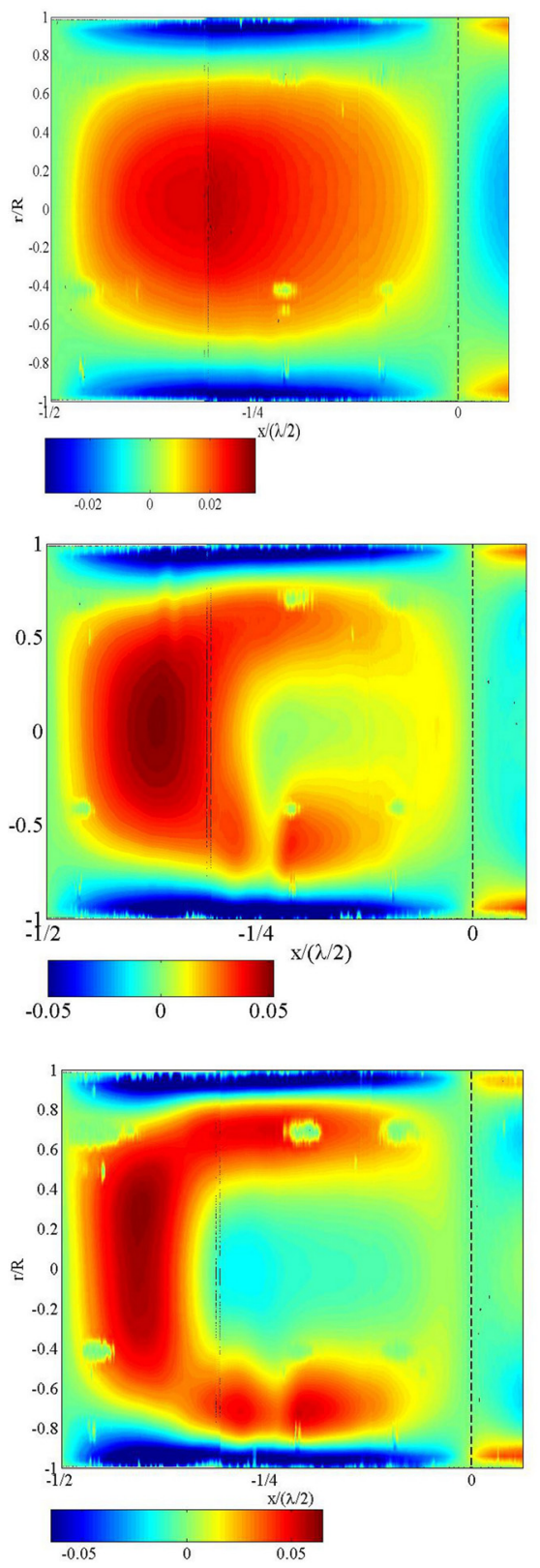
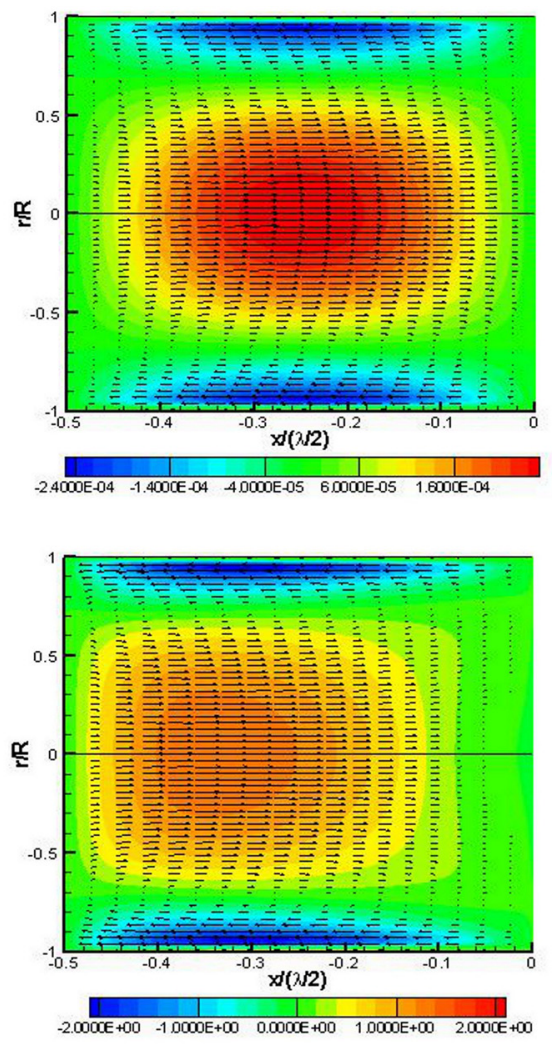

FIG. 3. (Color online) Contours of axial streaming velocity colored by its value (in $\mathrm{m} / \mathrm{s}$ ) and velocity vectors. Left: PIV results from $E_{2}$ for $\mathrm{Re}_{\mathrm{NL}}=$ $6,14,30$, respectively, from top to bottom, Right: numerical results from $\mathrm{N}_{3}$ for $\operatorname{Re}_{\mathrm{NL}}=0.006,51,111$, respectively, from top to bottom.
Figure 3 middle and bottom show the evolution of streaming when the acoustic level is increased. As found previously, the cell centers are first pushed towards the acoustic velocity nodes and then a counter-rotating cell appears along the resonator axis. ${ }^{9,10}$ PIV data are altered by light reflections on the tube glass but still display the $\mathrm{C}$-shape formed by the area of positive velocity (the yellow/red area). The change in the streaming pattern is not significant in the nearwall region. The position of the streaming velocity maximum in the near-wall region only shifts toward the acoustic velocity node as the acoustic level increases.

This presentation of the mutation of streaming at high levels is the one found in the literature. We need to consider it from another point of view in order to find its origin. In the following, in order to investigate the possible nonlinear interaction between streaming and acoustics and the associated validity of the method of successive approximations, we extract from the studied configurations presented in Table II a comparison of acoustic and streaming contribution to the different components of particle velocity at several strategic positions. In this section axial velocities are considered, Sec. IV being devoted to the investigation of radial velocities.

\section{B. Evolution of axial velocities}

Figure 4 shows acoustic and streaming axial velocities along the axis of the guide on a half wavelength span for two different acoustic levels extracted from $E_{2}$ set. An electric circuit equivalent to the electro-acoustic system has been developed that allows us to calculate the acoustic pressure and particle velocity along the resonator at any working frequency. Theoretical expectations obtained from this electroacoustic model are given for acoustic velocity and theoretical expectations for streaming from Rayleigh law [Eq. (1)] are also given. This figure makes it apparent that although the streaming is highly distorted at high amplitudes, the axial acoustic velocity keeps the same axial evolution. 

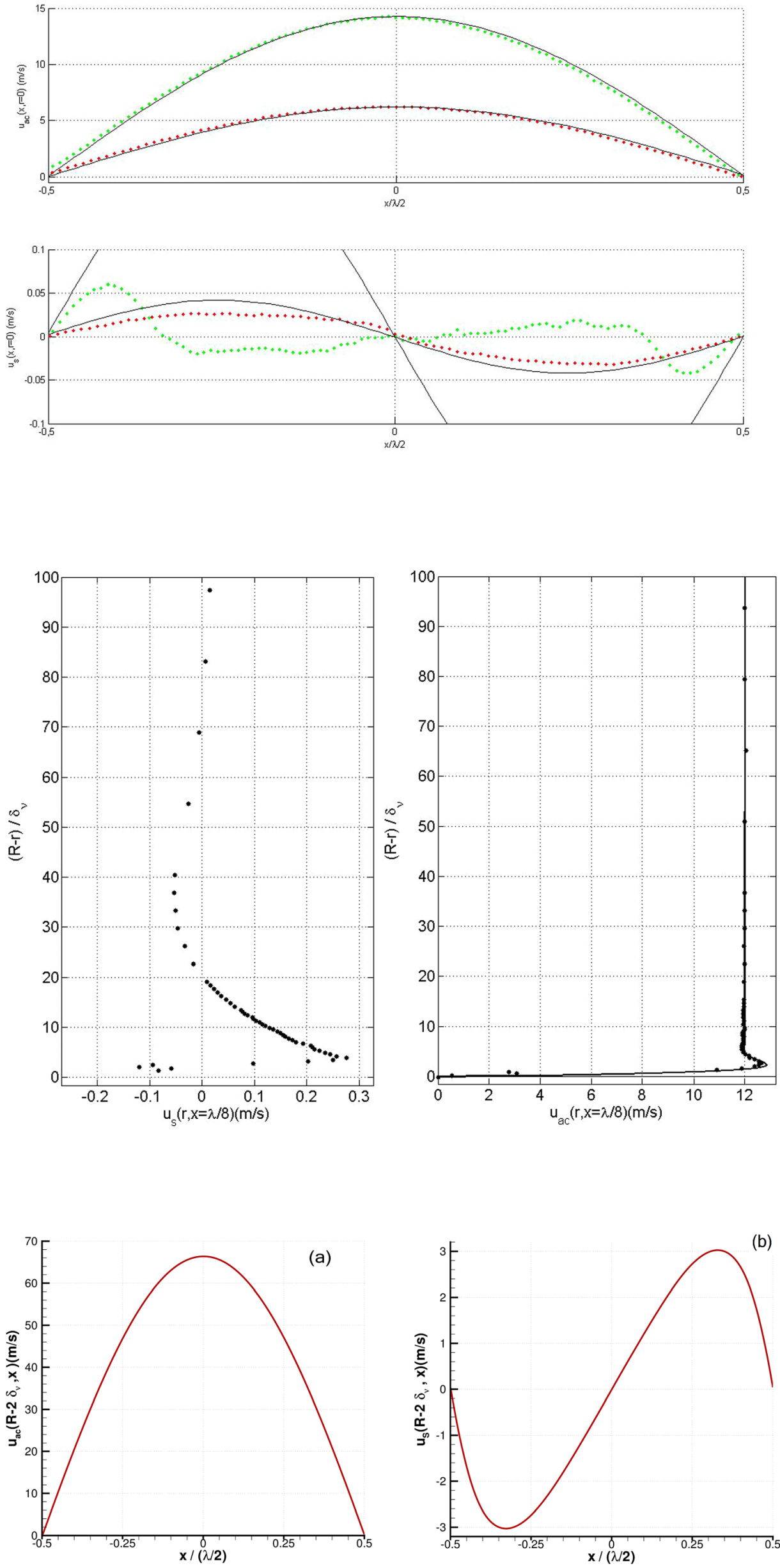

FIG. 4. (Color online) Top: axial acoustic velocity wave pattern: $u_{\mathrm{ac}}(x, r=0)$ in $\mathrm{m} / \mathrm{s}$ and bottom: axial streaming velocity along the guide axis: $u_{s}(x, r=0)$ in $\mathrm{m} / \mathrm{s}$ for low (red) and high (green) acoustic levels. Dots: experimental results from $E_{2}$. Lines: theoretical results from an electrodynamic model of the system (top) and from Eq. (1) (bottom).

FIG. 5. Left: radial dependence of axial acoustic streaming velocity: $u_{s}(x=\lambda / 8, r)$ in $\mathrm{m} / \mathrm{s}$. Right: radial dependence of axial acoustic velocity: $u_{\mathrm{ac}}(x=\lambda / 8, r)$ in $\mathrm{m} / \mathrm{s}$ for high acoustic level. Dots: LDV experimental results from $E_{2}$. Lines: theoretical expectation from Eq. (4).

FIG. 6. (Color online) (a) Axial acoustic velocity wave pattern: $u_{\mathrm{ac}}(x, r=$ $R-2 \delta_{\nu}$ ) in $\mathrm{m} / \mathrm{s}$ and (b) Axial streaming velocity: $u_{s}\left(x, r=R-2 \delta_{\nu}\right)$ in $\mathrm{m} / \mathrm{s}$ for high acoustic levels $\left(\operatorname{Re}_{\mathrm{NL}}=81\right)$. Numerical results from $N_{3}$. 


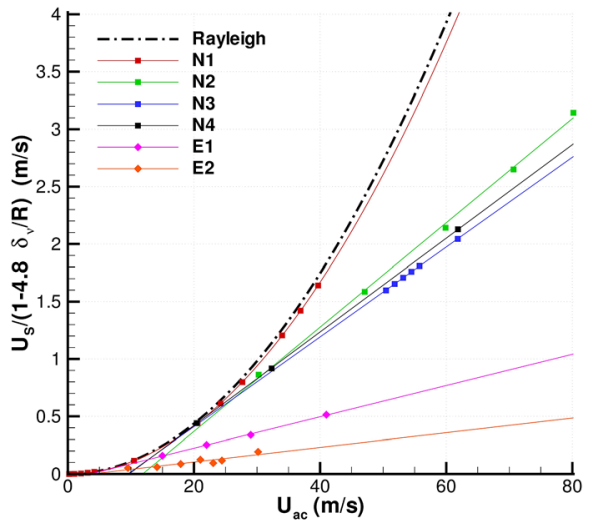

Similarly Fig. 5 shows acoustic and streaming axial velocities along the radial coordinates for an high acoustic level extracted from $E_{2}$ set. Theoretical expectation for axial acoustic velocity is also given. The latter is obtained from the integration of the first order axial component of NavierStokes equations, giving

$$
u_{\mathrm{ac}}(x, r)=\frac{1}{i \omega \rho_{0}} \frac{\partial p_{\mathrm{ac}}}{\partial x}\left(1-\frac{J_{0}(b r / R)}{J_{0}(b)}\right),
$$

with $b=(1+i) R / \delta_{\nu}, \rho_{0}$ the mean density of the fluid, $p_{\mathrm{ac}}$ the acoustic pressure and $J_{0}$ is the Bessel function of the first kind of order 0. Again this figure shows that although the axial streaming velocity has a radial dependence very different from a parabola as described in Eq. (1), the axial acoustic velocity keeps the same radial evolution even in the very near wall region where streaming could be expected to alter acoustics velocity due to severe velocity gradients encountered.

Numerical results confirm this evolution as shown by Fig. 6 where axial acoustic and streaming velocities are represented along a streaming cell in the near wall region: $u_{a c}\left(x, r=R-2 \delta_{\nu}\right)$ and $u_{s}\left(x, r=R-2 \delta_{\nu}\right)$, for high acoustic level. As already observed, the streaming pattern in this condition is distorted; however, the acoustic velocity is not modified.

\section{Relationship between streaming and acoustic velocities}

\section{Outer streaming}

In this section we further investigate the validity of Eq. (1) and particularly the dependence of the axial streaming velocity on the acoustic velocity for different configurations. Figure 7 is the result of a search of a relationship between the axial outer streaming velocity amplitude $U_{s}$ and the acoustic velocity amplitude $U_{\text {ac }}$. At low amplitude, the best fit with numerical and experimental results is found when representing the variation of $U_{s} \times\left\{1 /\left[1-4.8\left(\delta_{\nu} / R\right)\right]\right\}$ against $U_{\mathrm{ac}}$. Therefore, a dependence upon the aspect ratio $R / \delta_{\nu}$ should be included in Eq. (1) for slow streaming:

$$
U_{s} \propto U_{\mathrm{ac}}^{2}\left(1-4.8 \frac{\delta_{\nu}}{R}\right) .
$$

Figure 7 also shows that in each configuration the quadratic dependence given by Rayleigh's law Eq. (1) is only true up to
FIG. 7. (Color online) Axial streaming velocity amplitude $U_{s} \times[1 / 1-4.8$ $\left.\left(\delta_{\nu} / R\right)\right]$ against acoustic velocity amplitude $U_{\text {ac. }}$. Right: zoom on the low level region. a certain value of $U_{\mathrm{ac}}$. The dependence then becomes linear and this dependence is different for each configuration (different slopes of straight lines departing from the quadratic curve).

Figure 8 presents the same results as Fig. 7 but normalized with $U_{\text {Rayleigh }}=3 U_{\text {ac }}^{2} / 8 c$ and in a log-log scale. The ordinate is $U_{s} / U_{\text {Rayleigh }} \times\left\{1 /\left[1-4.8\left(\delta_{\nu} / R\right)\right]\right\}$ so that at low regime all curves start from 1 . It confirms that at low amplitudes the quadratic dependence is satisfied: All points fall on the horizontal line. The corresponding flow regime is thereafter called the first regime (Regime 1). Then a second regime (Regime 2) can be identified, for which all curves are of slope -1 on a $\log -\log$ scale.

There exist configurations in Regime 2 where the emergence of the extra cell has not been observed yet $\left(N_{4}\right)$. Therefore, there is no simple and direct correlation between the transition to the second regime and the appearance of a new cell.

\section{Inner streaming}

As for outer streaming, a relationship between the axial inner streaming velocity maximum $U_{s}$ and the acoustic velocity amplitude $U_{\text {ac }}$ is searched. The best fit results in a parabolic variation of $U_{s}\left(x_{m}, r=R-3 \delta_{\nu}\right) /\left[1-6.8\left(\delta_{\nu} / R\right)\right]$ against $U_{\text {ac }}$ as shown in Fig. 9. The coefficient 6.8 is different from the coefficient obtained for outer streaming because

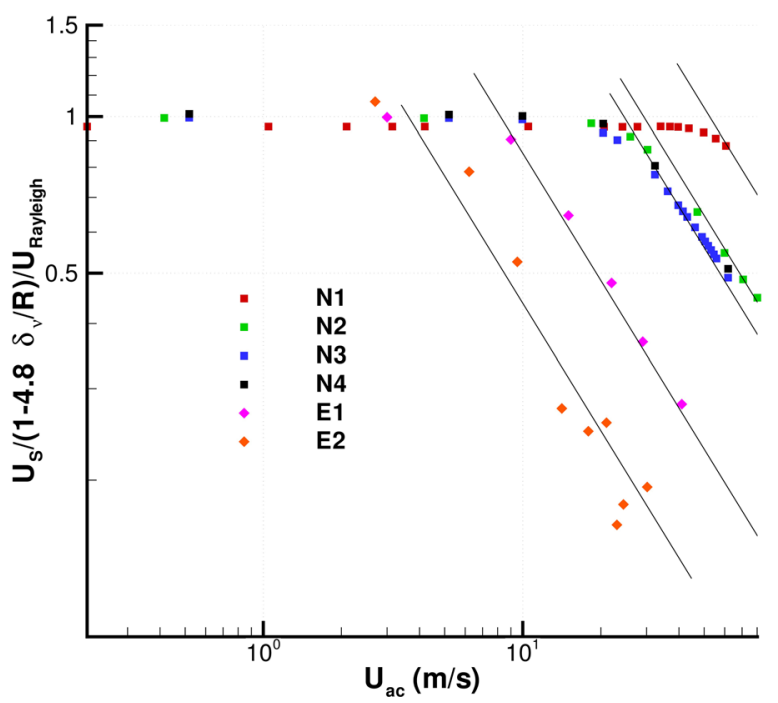

FIG. 8. (Color online) $\left(U_{s} / U_{\text {Rayleigh }}\right) \times\left[1 / 1-4.8\left(\delta_{\nu} / R\right)\right]$ against $U_{\text {ac }}$ on a $\log -\log$ scale. 

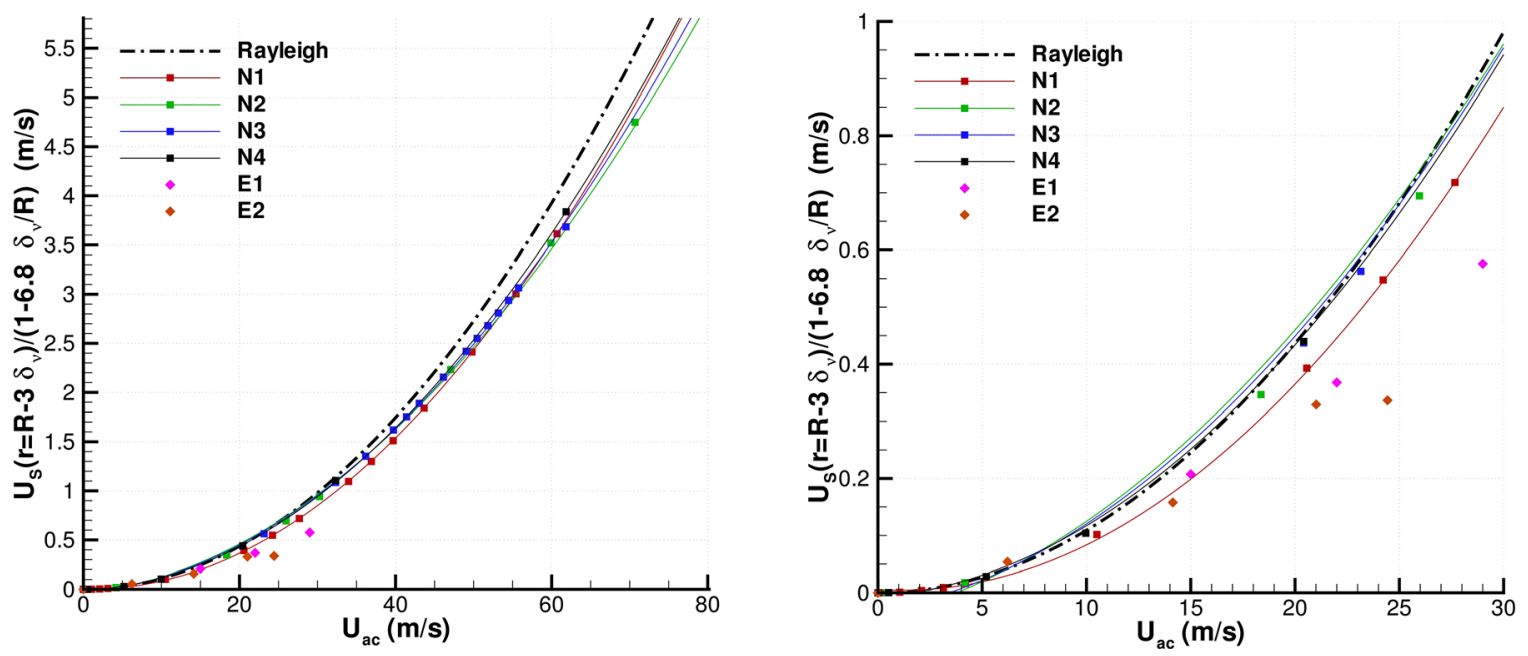

FIG. 9. (Color online) $U_{s} /\left[1-6.8\left(\delta_{\nu} / R\right)\right]$ against $U_{\text {ac }}$ within the near-wall region. Right: zoom for low levels.

the two streaming velocities are at two different radial positions, $r=R-3 \delta_{\nu}$ for inner streaming and $r=0$ for outer streaming. In the simulation, $x_{m}$ corresponds to the axial position of streaming velocity maximum at $r=R-3 \delta_{\nu}$ which is the place of inner streaming amplitude. All simulation points follow the same curve. Experimental data are taken in the near-wall region but it could not be precisely set at the same relevant position so the fit of experimental data is not obvious. However, in all cases it can be concluded that the quadratic dependence of inner streaming on acoustics still holds even at high amplitudes.

\section{RADIAL ACOUSTIC AND STREAMING VELOCITIES FROM LOW TO HIGH LEVELS}

Finally in this section the mutation of axial streaming velocity was further investigated and it was shown that there is no need to reconsider the application of the perturbation method for axial components of particle velocity. In Sec. IV A, the case of radial velocities is considered.

\section{A. Evolution of radial velocities}

The radial component of acoustic particle velocity can be obtained from the radial integration of the first order continuity equation (e.g., Ref. 16)

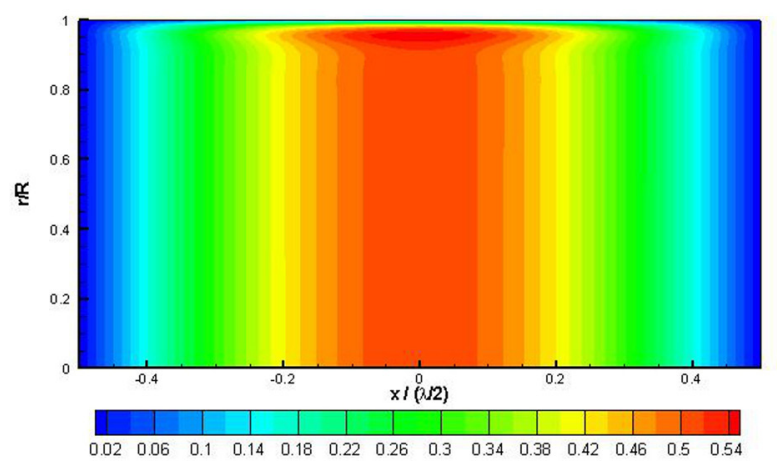

$$
\begin{aligned}
v_{\mathrm{ac}}(x, r)= & \frac{i R}{\omega \rho_{0}}\left[\frac{\partial^{2} p_{\mathrm{ac}}}{\partial x^{2}}\left(\frac{r}{2 R}-\frac{J_{1}(b r / R)}{b J_{0}(b)}\right)\right. \\
& \left.+\frac{\omega^{2}}{c^{2}} p_{\mathrm{ac}}\left(\frac{r}{2 R}+\frac{(\gamma-1) J_{1}(\sqrt{\sigma} b r / R)}{\sqrt{\sigma} b J_{0}(\sqrt{\sigma} b)}\right)\right],
\end{aligned}
$$

where $\sigma$ is the Prandtl number. This equation shows that the transverse acoustic velocity has the same axial dependence as the acoustic pressure, thus being maximal at axial positions of $u_{\mathrm{ac}}$ nodes in a standing wave pattern. Apart from the near wall region, $v_{\mathrm{ac}}$ varies linearly with $r$ [whereas $u_{\mathrm{ac}}$ is almost constant across the guide section, see Eq. (4)]. $v_{\mathrm{ac}}$ is zero on the guide axis and is anti-symmetric with respect to this axis. Finally $v_{\mathrm{ac}}$ owes its origins in the compressibility of the fluid and scales as $\left(\delta_{\nu} / \lambda\right) u_{\mathrm{ac}}$ (see Ref. 17). These characteristics are shown by Fig. 10 that compares colormaps for acoustic axial and radial components and by Fig. 11 that shows the transverse variation of acoustic radial velocity at different axial positions for low level, calculated from $N_{3}$, together with a linear fit. The corresponding axial positions, labeled from 1 to 6, are highlighted on Fig. 10, right.

Similarly, as shown by Eq. (2), the radial streaming velocity component has an axial dependence opposite to that of the axial streaming velocity, has an anti-symmetry across the axis of the guide where it is zero, and is much smaller than the axial streaming velocity. This is shown by Fig. 12.

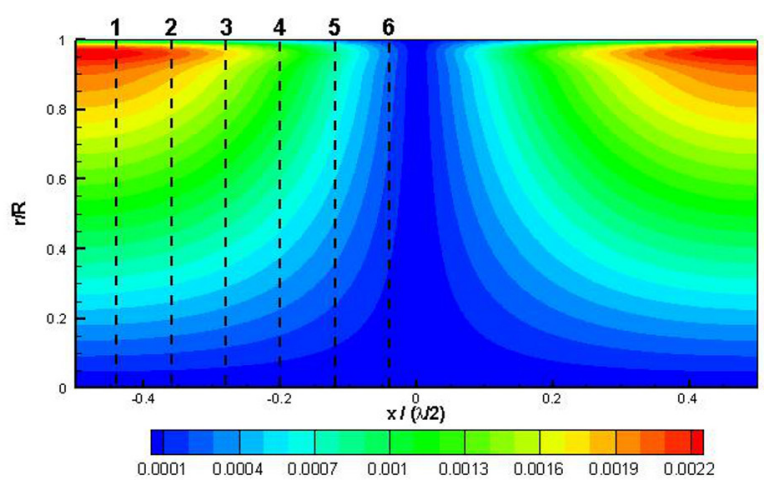

FIG. 10. (Color online) Contours of the axial acoustic velocity $u_{\mathrm{ac}}(\mathrm{m} / \mathrm{s})$ (left) and the radial acoustic velocity $v_{\text {ac }}(\mathrm{m} / \mathrm{s})$ (right) colored by its value obtained with the DNS simulation $\left(N_{3}\right), \mathrm{Re}_{\mathrm{NL}}=0.006$. 


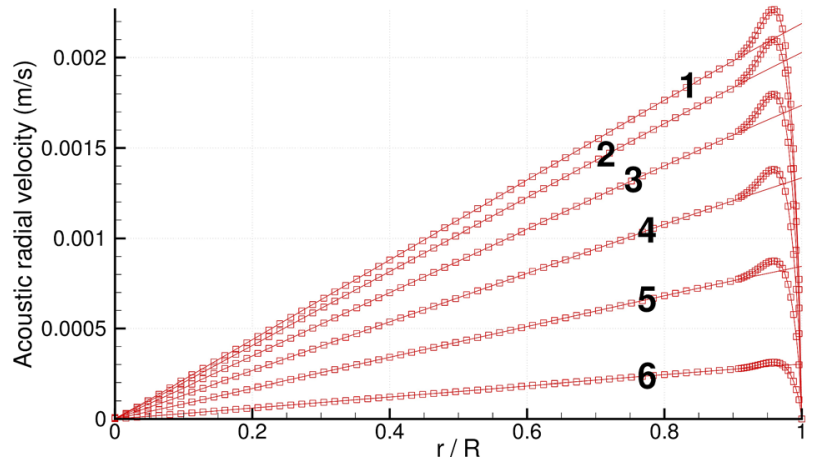

FIG. 11. (Color online) Radial dependence of radial acoustic velocity obtained from the DNS calculation at different axial locations: $v_{\mathrm{ac}}(-\lambda / 4+$ $m, r)$ with $m=3,7,11,15,19$, and $23 \times \lambda / 100$ for curves labeled, respectively, from 1 to 6 (Fig. 10, right) in $\mathrm{m} / \mathrm{s}$ for low acoustic level $\mathrm{Re}_{\mathrm{NL}}=0.006$. Squares: numerical results from $N_{3}$; Plain lines: linear fit.

In the region $x=0$, the radial streaming velocity $v_{s}$ is higher than the radial acoustic velocity $v_{\text {ac }}$.

The following figures show that the above listed low amplitude features of radial velocities are modified for both the acoustic and the streaming radial velocities as the acoustic amplitude is increased. Figure 13, left (similar to Fig. 10, right but for higher amplitude: $\mathrm{Re}_{\mathrm{NL}}=22$ ) shows that when the acoustic amplitude is increased, the radial acoustic velocity encounters significant changes. Figure 13, right shows the radial variation of the radial acoustic velocity $v_{\mathrm{ac}}(r)$ obtained at different axial positions (labeled from 1 to 6 , highlighted on Fig. 13, left). In the region around $x=0$ (curve labeled 6), the profile is no longer linear in $r$, but is a polynomial of the 3rd order, as found by a polynomial fit in the core region. Therefore, it appears that even if the axial acoustic velocity $u_{\mathrm{ac}}$ is not modified [neither in its axial variation $u_{\mathrm{ac}}(x)$ nor in its radial variation $\left.u_{\mathrm{ac}}(r)\right]$ when the acoustic amplitude is increased, the radial acoustic velocity $v_{\text {ac }}$ is modified.

Figure 14 shows the corresponding evolution of radial streaming velocity. $v_{s}(x, r=0.564 R)$ is no longer sinusoidal [see Fig. 14(c)] and the symmetry between $v_{s}(x=0, r)$ and $v_{s}(x=\lambda / 2, r)$ is no longer observed, with the radial streaming velocity around $x=0$ globally decreasing [see Fig. 14(b)]. In this region, however, $v_{s}$ is still higher than $v_{\text {ac }}$.
Let us now consider the case $N_{3}, \operatorname{Re}_{\mathrm{NL}}=81$, which corresponds to a case in Regime 2 with an extra counterrotating cell. Figure 15 displays the radial acoustic velocity obtained from the DNS calculation. The profiles in the core are very distorted and correctly fitted by a polynomial of 6th order. Only in the $x= \pm \lambda / 4$ regions does the radial acoustic velocity keep a linear radial variation. In addition, as shown by Fig. 16, when the acoustic level is increased, the radial streaming velocity in the $x=0$ region continues to decrease until tending to zero and then changing sign, in association with the generation of the additional outer streaming cell.

Finally, both the radial acoustic velocity and the radial streaming velocity are subject to important changes as the acoustic level is increased. Due to the fact that the modification of the radial acoustic velocity first occurs in the region where the radial streaming velocity is large, it can be hypothesized that streaming alters acoustics. This possibility is investigated in Sec. IV B.

\section{B. Nonlinear interaction of radial acoustic and streaming velocities}

In order to assess the interaction between acoustic and streaming flows, a DNS calculation is conducted where at the end of each period, the averaged flow over the preceding period is subtracted from the total instantaneous velocity field, before starting a new time step. By doing so, we intend to artificially remove the influence of the streaming flow on the acoustic field. Figure 17 shows the radial variation of the resulting radial acoustic velocity component $v_{\mathrm{ac}}(r)$ for the case discussed in Sec. IV A $\left(N_{3}, \mathrm{Re}_{\mathrm{NL}}=81\right.$, see Fig. 15). In the core region, the profile remains linear whereas the acoustic field extracted from the full DNS calculation had a radial component with a 6 th order polynomial variation in the radial direction (see Fig. 15, right). This observation proves that streaming flow influences the acoustic field through the radial component.

This is confirmed when looking back at the radial variation of the axial streaming velocity $u_{s}(r)$ and comparing results for the full DNS calculation with those obtained after having removed the influence of streaming on acoustics. The left part of Fig. 18 shows the radial variation of the axial streaming velocity calculated with full DNS. The profiles in the core are correctly fitted by a polynomial of 5 th order. On

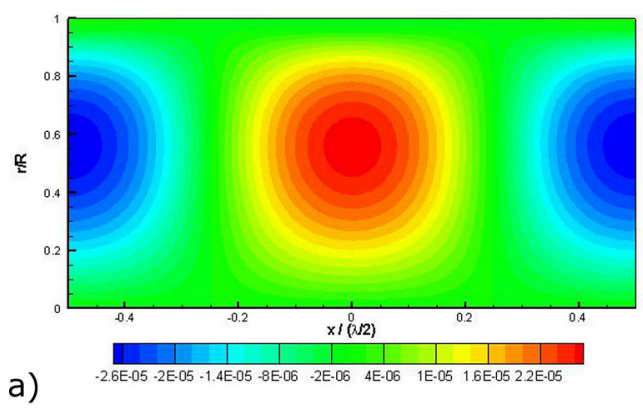

b)

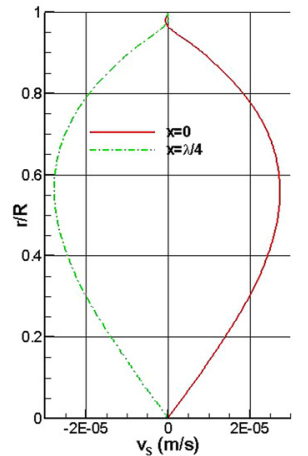

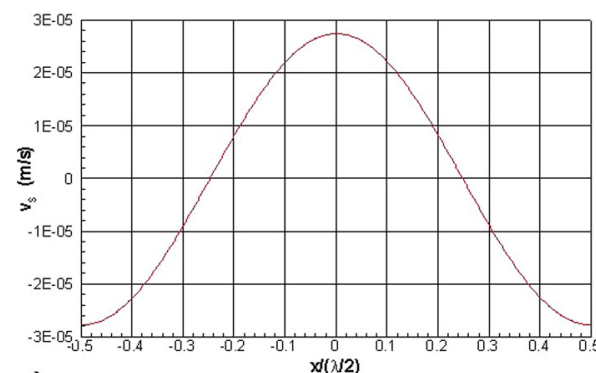

C)

FIG. 12. (Color online) (a) Contours of the radial streaming velocity $v_{s}$ colored by its value obtained with the DNS simulation. (b) Radial dependence of radial streaming velocity: $v_{s}(x=0, r)$ and $v_{s}(x=\lambda / 4, r)$ in $\mathrm{m} / \mathrm{s}$. (c) Axial dependence of radial streaming velocity: $v_{s}(x, r=0.564 R)$ in $\mathrm{m} / \mathrm{s}$. Numerical results from $N_{3}, \operatorname{Re}_{\mathrm{NL}}=0.006$ 

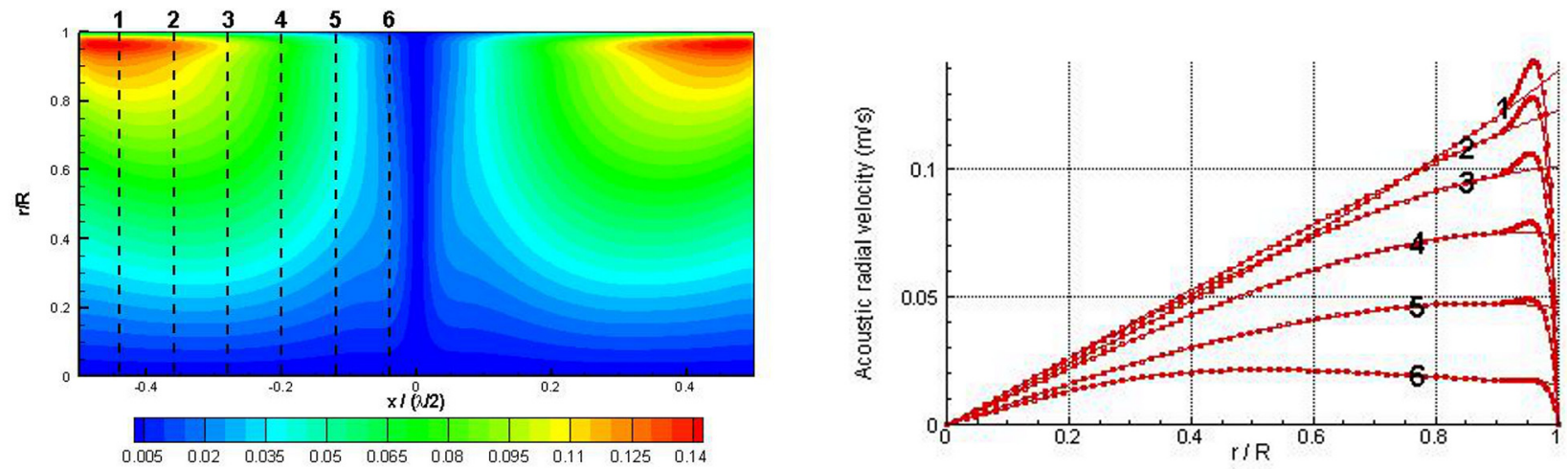

FIG. 13. (Color online) Left: contours of the radial acoustic velocity $v_{\text {ac }}$ amplitude colored by its value. Right: radial variation of the radial acoustic velocity obtained from the full DNS calculation at different axial locations $v_{\mathrm{ac}}(-\lambda / 4+m, r)$ with $m=3,7,11,15,19$, and $23 \times \lambda / 100$ for curves labeled, respectively, from 1 to 6 (see left figure), in $\mathrm{m} / \mathrm{s}, N_{3}, \mathrm{Re}_{\mathrm{NL}}=22$. Solid lines: polynomial fit in the core region.

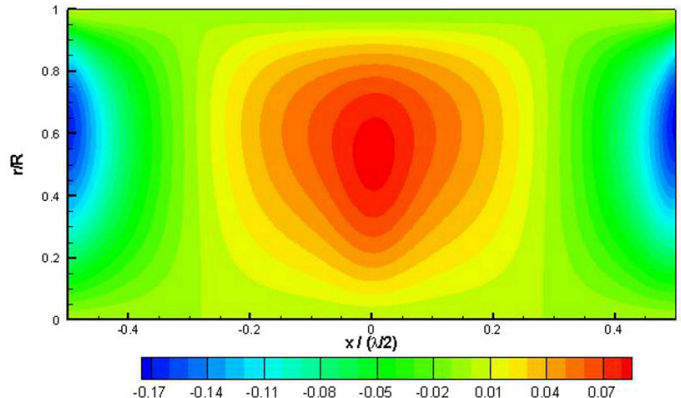

a)

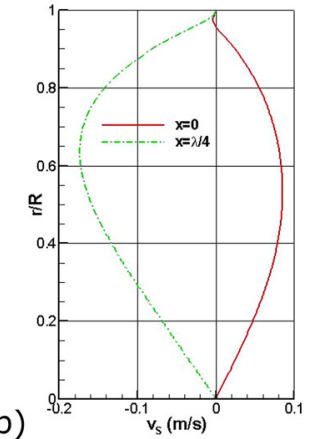

b)

$v_{s}(m / s)$

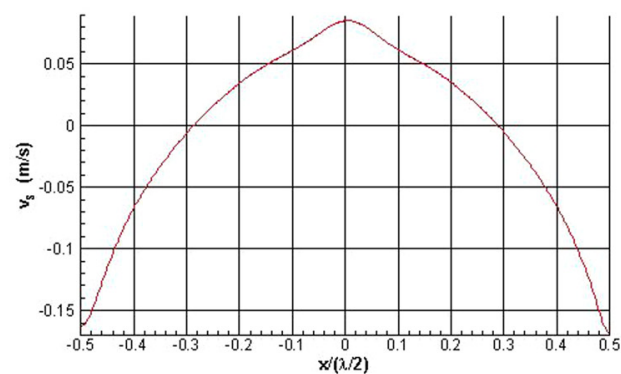

C)

FIG. 14. (Color online) (a) Contours of the radial streaming velocity $v_{s}$ colored by its value obtained with the DNS simulation. (b) Radial dependence of radial streaming velocity: $v_{s}(x=0, r)$ and $v_{s}(x=\lambda / 4, r)$ in $\mathrm{m} / \mathrm{s}$. (c) Axial dependence of radial streaming velocity: $v_{s}(x, r=0.564 R)$ in $\mathrm{m} / \mathrm{s}$. Numerical results from $N_{3}, \mathrm{Re}_{\mathrm{NL}}=22$.
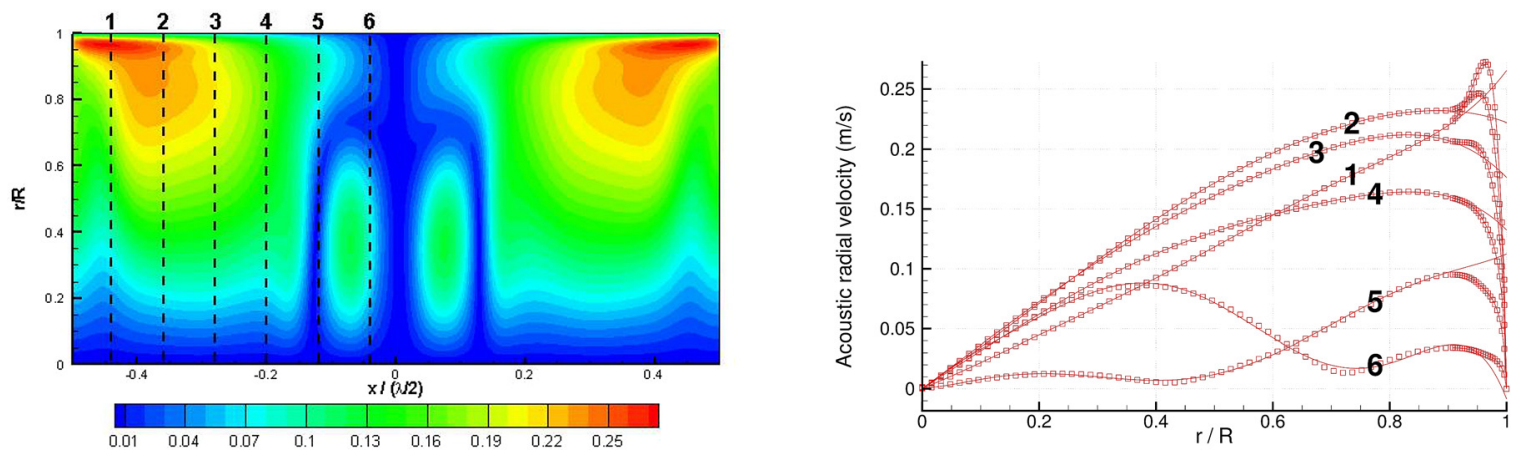

FIG. 15. (Color online) Left: contours of the radial acoustic velocity $v_{\text {ac }}$ amplitude colored by its value. Right: radial variation of the radial acoustic velocity obtained from the full DNS calculation at different axial locations $v_{\mathrm{ac}}(-\lambda / 4+m, r)$ with $m=3,7,11,15,19$, and $23 \times \lambda / 100$ for curves labelled, respectively, from 1 to 6 (see left figure) in $\mathrm{m} / \mathrm{s}, N_{3}, \mathrm{Re}_{\mathrm{NL}}=81$. Solid lines: polynomial fit in the core region.

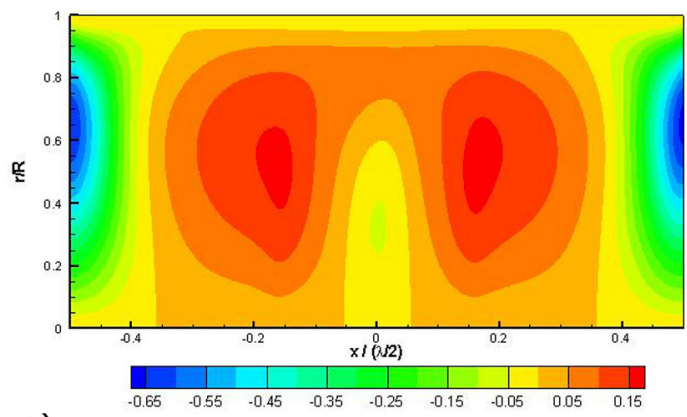

a)

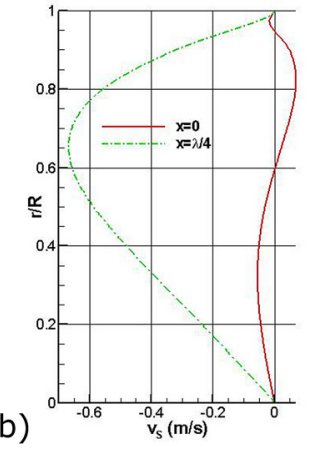

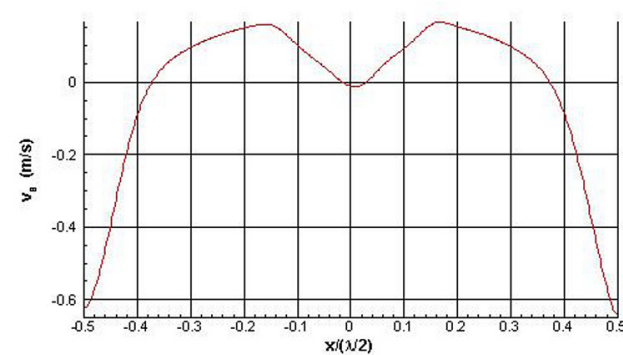

c)

FIG. 16. (Color online) (a) Contours of the radial streaming velocity $v_{s}$ colored by its value obtained with the DNS simulation. (b) Radial dependence of radial streaming velocity: $v_{s}(x=0, r)$ and $v_{s}(x=\lambda / 4, r)$ in $\mathrm{m} / \mathrm{s}$. (c) Axial dependence of radial streaming velocity: $v_{s}(x, r=0.564 R)$ in $\mathrm{m} / \mathrm{s}$. Numerical results from $N_{3}, \operatorname{Re}_{\mathrm{NL}}=81$. 


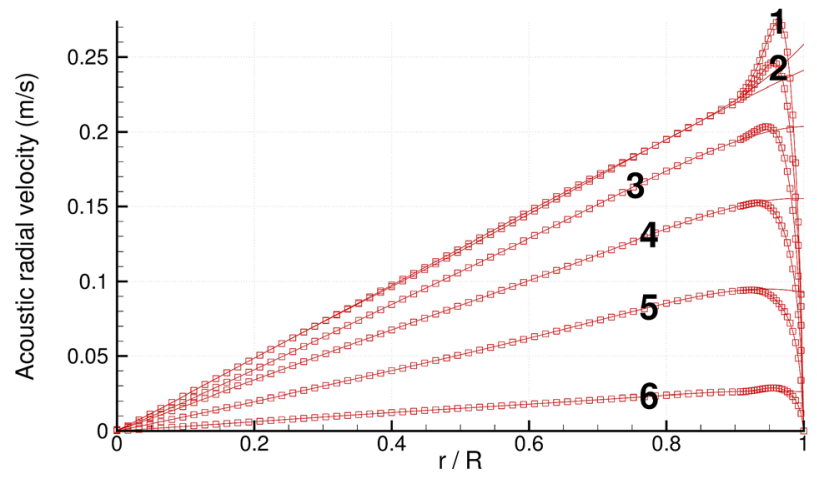

FIG. 17. (Color online) Radial variation of the radial acoustic velocity at different axial locations $v_{a c}(-\lambda / 4+m, r)$ with $m=3,7,11,15,19$, and $23 \times \lambda / 100$ for curves labeled, respectively, from 1 to 6 , in $\mathrm{m} / \mathrm{s}$ when removing the influence of the streaming flow on the acoustic flow, $N_{3}$, $\mathrm{Re}_{\mathrm{NL}}=81$. Solid lines: linear fit in the core region.

one of the profiles we can see negative values of the axial streaming velocity in the core, assessing the presence of the extra counter-rotating outer streaming cell. Now if the interaction between streaming and acoustics is removed, the right part of Fig. 18 shows the profiles are parabolic as shown by a polynomial fit and no extra cell is observed since the velocities are all positive in the core. The associated streaming pattern has a similar structure as a Regime 1 streaming flow pattern although created by a high intensity acoustic wave. This shows the major role played by the coupling between the streaming flow and the acoustic field. This coupling works as a two-way interaction through the radial components.

Let us now explore the relative amplitude of the radial acoustic and streaming velocity components. The left part of Fig. 19 shows the maximum absolute value of radial streaming velocity (empty symbols) and radial acoustic velocity (full symbols) as functions of the axial acoustic velocity amplitude $U_{\text {ac }}$ for the sets $N_{1}$ to $N_{3}$. One can observe that the radial acoustic velocity amplitude $V_{\text {ac }}$ depends linearly on $U_{\text {ac }}$ and is independent of $R / \delta_{\nu}$, in agreement with Eq. (6). The radial streaming velocity amplitude $V_{s}$ is a quadratic function of $U_{\mathrm{ac}}$, in agreement with Eq. (2). The amplitude of $v_{s}$ increases with $R / \delta_{\nu}$. After the intersection of the two sets of curves, $V_{s}$ becomes greater than $V_{\text {ac }}$, which occurs for sets
$N_{2}$ and $N_{3}$ but not for $N_{1}$. This is consistent with the transition from the first to the second regime of streaming described previously: The value of $U_{\text {ac }}$ corresponding to $V_{s}$ becoming greater than $V_{\text {ac }}$ in Fig. 19 is the same as the value of $U_{\mathrm{ac}}$ corresponding to the change of regime (from quadratic to linear) in Fig. 8. The right part of Fig. 19 shows similar results for the experimental configuration E2. Here the amplitude of streaming velocity was estimated from PIV measurements and the dependency of radial acoustic velocity on axial acoustic velocity was obtained by using Eq. (6). As was the case for numerical results (left part of Fig. 19), the value of $U_{\text {ac }}$ corresponding to $V_{s}$ becoming greater than $V_{\mathrm{ac}}$ is close to the value in Fig. 8 corresponding to the change of regime (from quadratic to linear). The quadratic dependency of $V_{s}$ on $U_{\mathrm{ac}}$ is verified experimentally. As was the case for $U_{s}$ (see Fig. 7), $V_{s}$ is close to Rayleigh's law Eq. (2) only for low acoustic levels. However, we do not observe here any change of regime for $V_{s}$ as was discussed for $U_{s}$ : Even if $V_{s}$ diverges from Eq. (2), it still depends quadratically on $U_{\mathrm{ac}}$.

Finally these results confirm that the strong nonlinear relation between the different velocity components for acoustics and streaming is responsible for the mutation of streaming patterns at high amplitude.

\section{CONCLUSION}

A study including numerical and experimental results was presented in order to analyse the different streaming flow regimes in a cylindrical waveguide. Two different regimes were identified. The first regime, Regime 1, corresponds to low acoustic amplitudes for which the axial streaming velocity amplitude is a quadratic function of the acoustic amplitude. In the second (high amplitudes) regime, Regime 2, streaming velocity becomes a linear function of the acoustic amplitude. A nonlinear interaction between the radial component of acoustic streaming and the radial component of acoustic velocity is responsible for this change of regime that occurs when the radial streaming velocity becomes larger than the radial acoustic velocity. This interaction then yields the generation of additional outer streaming cells. The axial and radial variations of the axial acoustic velocity remain the same when the acoustic level is
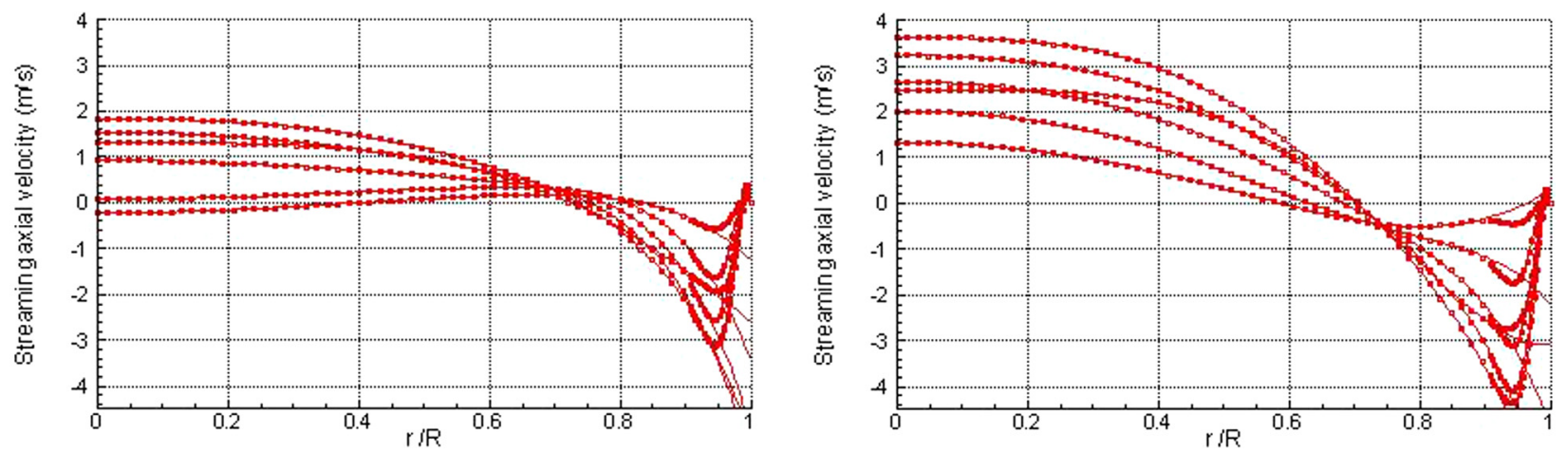

FIG. 18. (Color online) Radial variation of the axial streaming velocity induced by the acoustic flow shown in Fig. 15 (left: full DNS simulation) and 17 (right:when removing the influence of streaming on acoustics), $u_{\mathrm{ac}}(x=3,7,11,15,19$, and $23 \times \lambda / 100, r), N_{3}$, Re $\mathrm{NL}_{\mathrm{NL}}=81$. Solid lines: polynomial fit in the core region. 


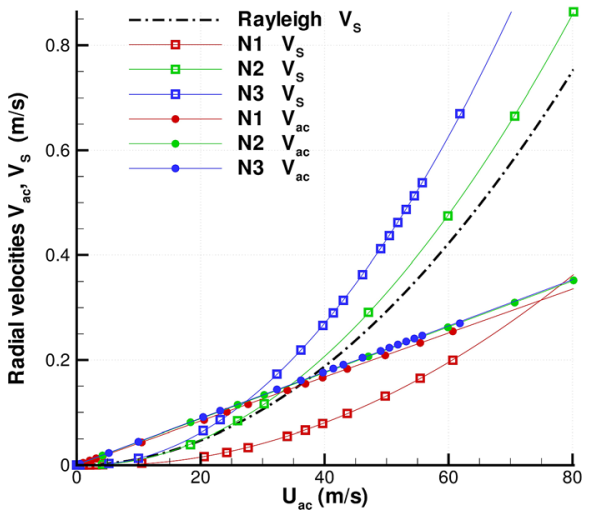

increased. On the contrary, the radial acoustic velocity and both the axial and radial streaming velocities are very different from low level velocities.

${ }^{1}$ L. Rayleigh, "On the circulation of air observed in Kundt's tubes, and on some allied acoustical problems," Philos. Trans. R. Soc. London 175, 1-21 (1884).

${ }^{2}$ H. Schlichting, "Berechnung ebener periodischer grenzschichtströmungen (Calculation of plane periodic boundary layer streaming)," Phys. Z. 33(8), 327-335 (1932).

${ }^{3}$ Q. Qi, "The effect of compressibility on acoustic streaming near a rigid boundary for a plane traveling wave," J. Acoust. Soc. Am. 94, 1090-1098 (1993).

${ }^{4} \mathrm{~N}$. Rott, "The influence of heat conduction on acoustic streaming," $\mathrm{Z}$. Angew. Math. Phys. (ZAMP) 25, 417-421 (1974).

${ }^{5}$ R. L. Smith and G. W. Swift, "Measuring second-order time-average pressure," J. Acoust. Soc. Am. 110(2), 717-723 (2001).

${ }^{6}$ J. Sharpe, C. Greated, C. Gray, and D. M. Campbell, "The measurements of acoustic streaming using particle image velocimetry," Acta Acust united Ac 68(2), 168-172 (1989).

${ }^{7}$ M. Thompson and A. Atchley, "Simultaneous measurement of acoustic and streaming velocities in a standing wave using laser Doppler anemometry," J. Acoust. Soc. Am. 117, 1828-1838 (2005).

${ }^{8}$ S. Moreau, H. Bailliet, and J.-C. Valière, "Measurements of inner and outer streaming vortices in a standing waveguide using laser Doppler velocimetry," J. Acoust. Soc. Am. 123, 640-647 (2008).
FIG. 19. (Color online) Maximum radial streaming (empty symbols) and acoustic (full symbols) velocities versus $U_{\text {ac }}$ (maximum axial acoustic amplitude). Left: numerical results. Right: experimental results [using Eq. (6) for an estimate of $V_{\mathrm{ac}}$.
${ }^{9}$ I. Reyt, H. Bailliet, and J.-C. Valière, "Experimental investigation of acoustic streaming in a cylindrical waveguide up to high streaming Reynolds numbers,” J. Acoust. Soc. Am. 135, 27-37 (2014).

${ }^{10}$ I. Reyt, V. Daru, H. Bailliet, S. Moreau, J.-C. Valière, D. Baltean-Carlès, and C. Weisman, "Fast acoustic streaming in standing waves: Generation of an additional outer streaming cell," J. Acoust. Soc. Am. 134, 1791-1801 (2013).

${ }^{11}$ L. Menguy and J. Gilbert, "Non-linear acoustic streaming accompanying a plane stationary wave in a guide," Acta Acust united Ac 86(2), 249-259 (2000).

${ }^{12} \mathrm{~S}$. Boluriaan and P. J. Morris, "Numerical simulation of acoustic streaming in high amplitude standing wave," 9th AAIA/CEAS Aeroacoustics Conference, Hilton Head, SC, AIAA Paper N²003-3152 (12-14 May 2003).

${ }^{13}$ V. Daru, D. Baltean-Carlès, and C. Weisman, "Inertial effects on nonlinear acoustic streaming," AIP Conf. Proc. 1685, 030003 (2015).

${ }^{14}$ M. Thompson, A. Atchley, and M. Maccarone, "Influences of a temperature gradient and fluid inertia on acoustic streaming in a standing wave," J. Acoust. Soc. Am. 117, 1839-1849 (2005).

${ }^{15}$ V. Daru, D. Baltean-Carlès, C. Weisman, P. Debesse, and G. Gandikota, "Two-dimensional numerical simulations of nonlinear acoustic streaming in standing waves," Wave Motion 50, 955-963 (2013).

${ }^{16}$ H. Bailliet, V. Gusev, R. Raspet, and R. A. Hiller, "Acoustic streaming in closed thermoacoustic devices," J. Acoust. Soc. Am. 110, 1808-1821 (2001).

${ }^{17}$ N. Sugimoto, "Nonlinear theory for thermoacoustic waves in a narrow channel and pore subject to a temperature gradient," J. Fluid Mech. 797, 765-801 (2016). 